\title{
Rot-Buchen im Palmengarten - ein Kurzportrait
}

\author{
Katharina Kamm
}

\begin{abstract}
Common beech forests are the potential natural vegetation of Middle Europe. The areal of the common beech (Fagus sylvatica) reaches the Black Sea in the south east. Morphology and ecology of the common beech is shortly presented. In addition four different cultivars of the common beech are shown: Fagus sylvatica 'Zlatia', Fagus sylvatica 'Pendula', Fagus sylvatica f. purpurea and Fagus sylvatica 'Asplenifolia'.
\end{abstract}

\section{Zusamenfassung}

Buchenwälder bilden in großen Teilen Mitteleuropas die potenziell natürliche Vegetation. Das Verbreitungsgebiet der Rot-Buche (Fagus sylvatica) reicht im Südosten bis zum Schwarzen Meer. Morphologie und Ökologie der Rot-Buche werden kurz vorgestellt. Außerdem werden vier verschiedene Kultivare der Rot-Buche aus dem Palmengarten beschrieben: Fagus sylvatica 'Zlatia', Fagus sylvatica 'Pendula', Fagus sylvatica f. purpurea und Fagus sylvatica 'Asplenifolia'.

\section{Buchenwälder dominieren bei uns}

Die Rot-Buche (Fagus sylvatica) ist der einzige Vertreter der Gattung Fagus in unseren heimischen Wäldern. Im Jahr 2012 hatten Buchen einen Anteil von über $15 \%$ an der Waldfläche Deutschlands (bwi.info), das entspricht etwa $5 \%$ der Fläche der Bundesrepublik. Potenziell wären jedoch zwei Drittel Deutschlands

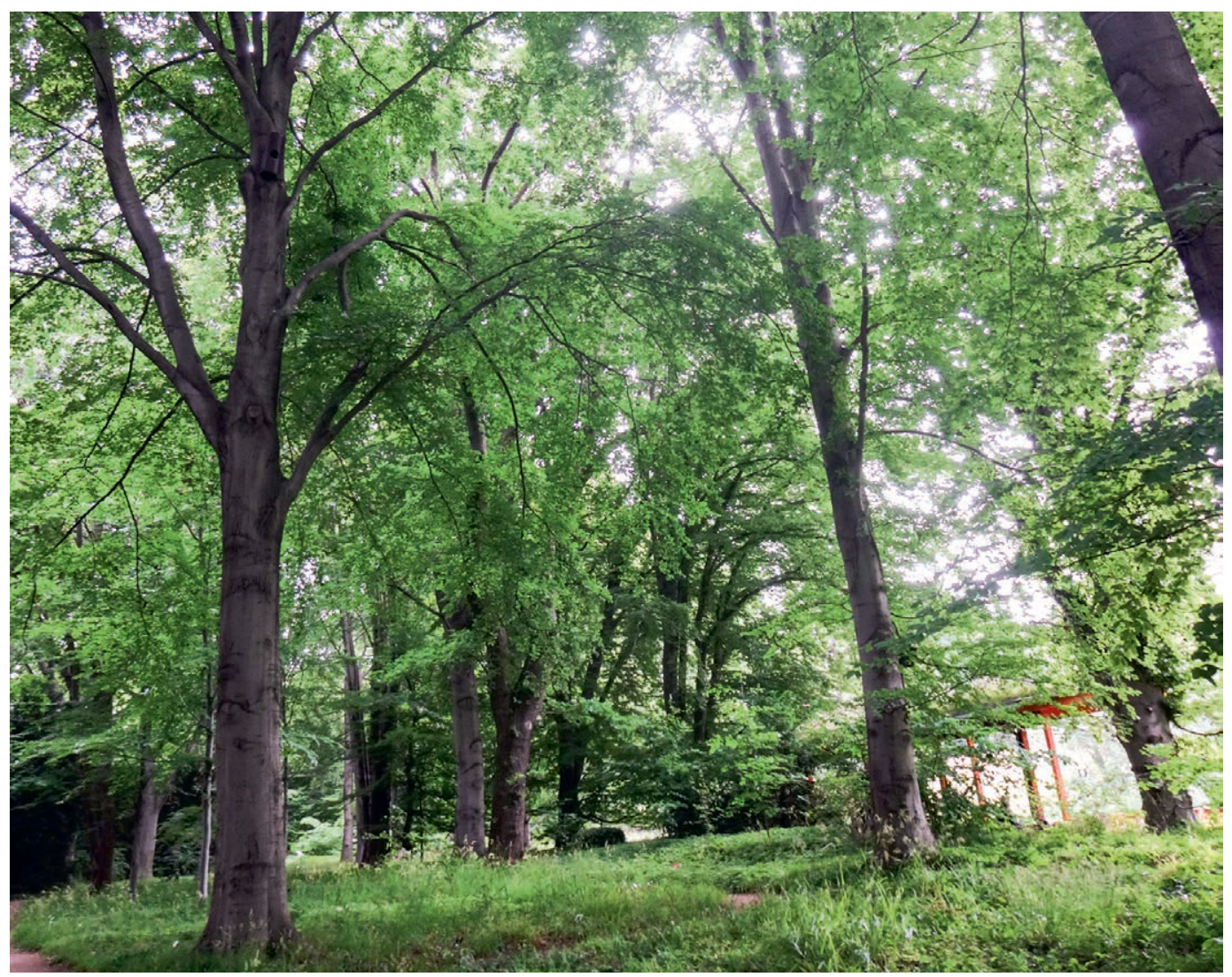

Abb. 1: Buchenmischwald im Botanischen Garten Frankfurt. (Foto: K. Kaмm) 
natürlicherweise Buchen(misch)wald (BоHN \& Neuhäusl 2000/2003). Dies ist ein guter Grund, sich diese Baumart einmal etwas genauer anzusehen.

\section{Verbreitung}

Das große potenzielle Verbreitungsgebiet der RotBuche in Deutschland ist auf ihre Konkurrenzstärke zurückzuführen. Durch ihre hohe Schattentoleranz können sich junge Rot-Buchen unter dicht geschlossenen Kronendächern entwickeln, während lichtbedürftigere Arten unterdrückt werden (Felbermeier \& Mosandl 2002). Daher dominieren Rot-Buchen auf (auch für andere einheimische Bäume) optimalen Standorten. Erst wenn es für sie zu trocken, zu nass oder zu nährstoffarm und sauer wird, können andere Baumarten neben ihr bestehen und sie schließlich ersetzen. In Buchenwäldern wird durch die Beschattung die Krautschicht meist unterdrückt, wohingegen es eine dichte Krautschicht aus Frühblühern und Waldkräutern geben kann, welche man beispielsweise im Botanischen Garten Frankfurt zu bewundern vermag (Wittig \& Streit 2004).

Rot-Buchen kommen allerdings nicht nur in Deutschland vor. Ihr Verbreitungsgebiet reicht von Süd-Skandinavien bis Sizilien, von Nord-Spanien bis zum Schwarzen Meer. Unterbrochen und begrenzt wird ihre Verbreitung unter anderem durch die geringe Dürre- und (Spät-)Frostresistenz ihrer Jungbäume (Felbermeier \& Mosandl 2002, StinglWAgner et al. 2009).

\section{Merkmale und Verwandtschaft}

Rot-Buchen erreichen ein Alter von 160-300, im Extremfall 900 Jahren. Ihre Umtriebszeit, d. h. ihr Alter zum Zeitpunkt des Fällens im Rahmen der Forstwirtschaft liegt jedoch bei 80-150 Jahren (Felbermeier \& Mosandl 2002, StinglwaGNER et al. 2009).

Rot-Buchen können eine Höhe von $45 \mathrm{~m}$ und einen Stammdurchmesser von 2,9 m erreichen und bilden ein Herzwurzelsystem. Sie sind im Freistand tief beastet, im Forst aber oft bis zu $25 \mathrm{~m}$ astfrei (Felbermeier \& Mosandl 2002, Stinglwagner et al. 2009).

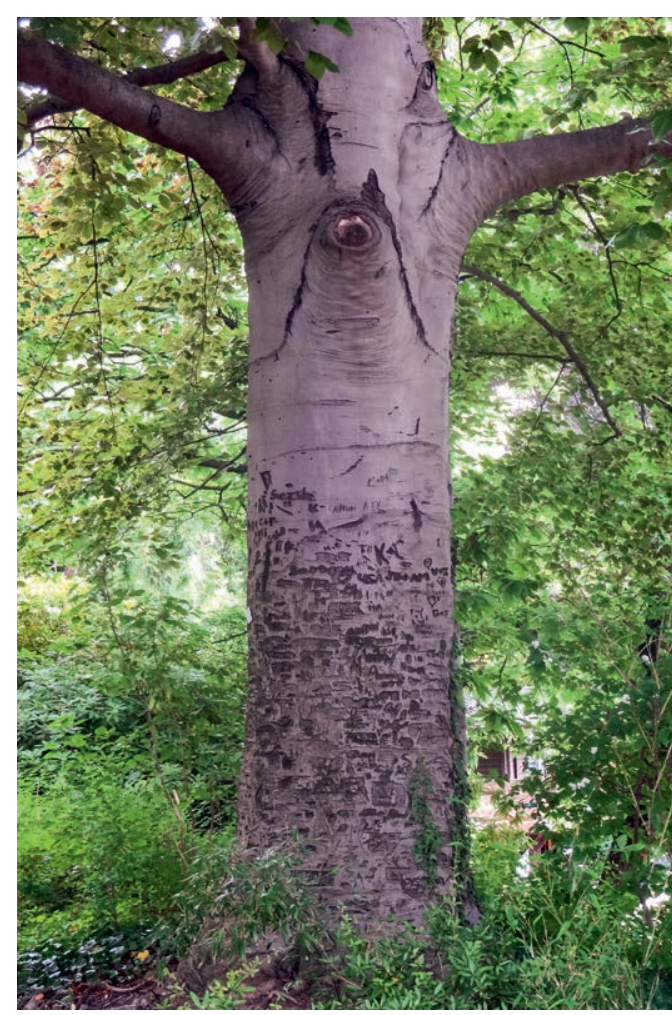

Abb. 2: Stamm einer Blut-Buche (Fagus sylvatica f. purpurea) mit Astnarbe und Einritzungen. (Foto: K. КАмм)

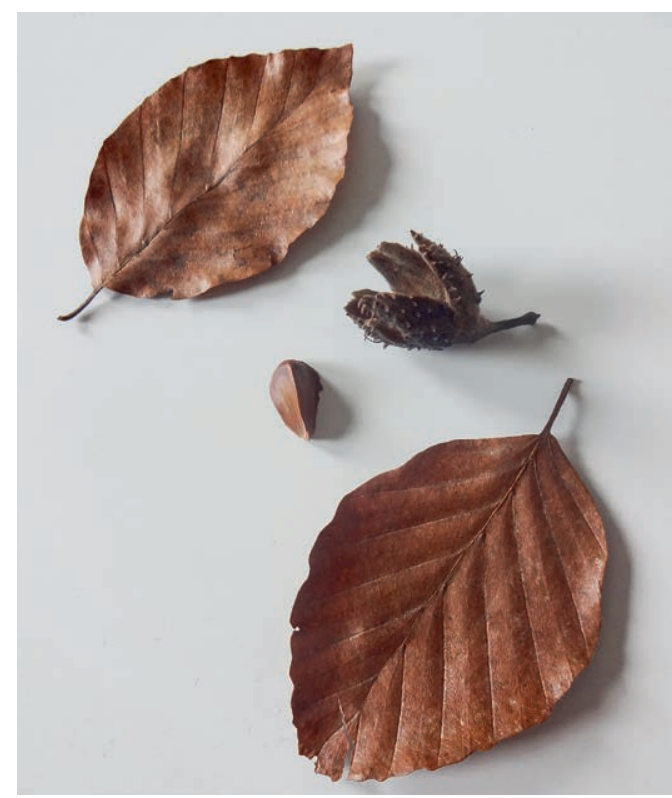

Abb. 3: Buchenlaub, Cupula und Buchecker. (Foto: K. Камм) 


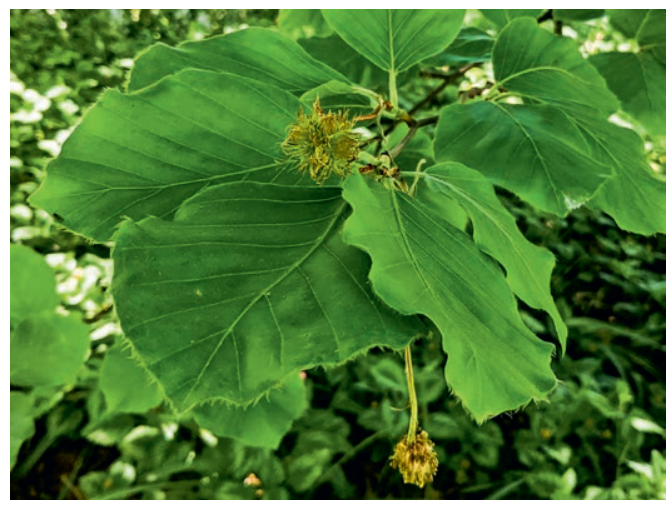

Abb. 4: Blätter, weibliche und männliche Blütenstände an einer Goldbuche. Fagus sylvatica 'Zlatia' (Foto: K. Камм)

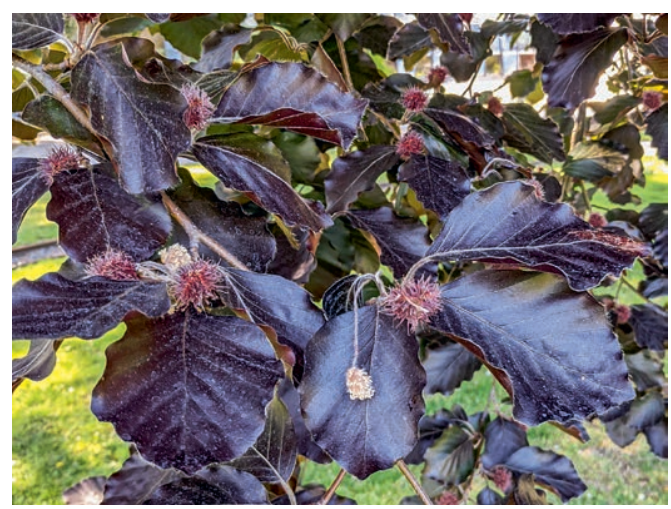

Abb. 5: Blätter, sowie weibliche und männliche Blütenstände an einer Blut-Buche. (Foto: K. Камм)

Ihre Rinde ist grau und glatt (SЕYвоLd 2006) und bleibt es auch meist bis ins hohe Alter, da bei Rot-Buchen neue Korkzellen etwa im Maße ihrer Abtragung gebildet werden. Die daher deutlich sichtbaren Astnarben setzen sich bei Rot-Buchen aus einer Rundnarbe und einer darüber gebogenen Winkelnarbe zusammen. (FELBERMEIER \& Mosandl 2002, StinglWagner et al. 2009).

Die glatte Rinde verleitet bisweilen dazu, Namen, Herzen und ähnliches in sie einzuritzen, was im schlimmsten Fall Pilzsporen und anderen Krankheitserregern Einlass in den Baum gewährt. Die Blätter der Rot-Buchen sind einfach, eiförmig zugespitzt und unterseits stellenweise behaart. Am

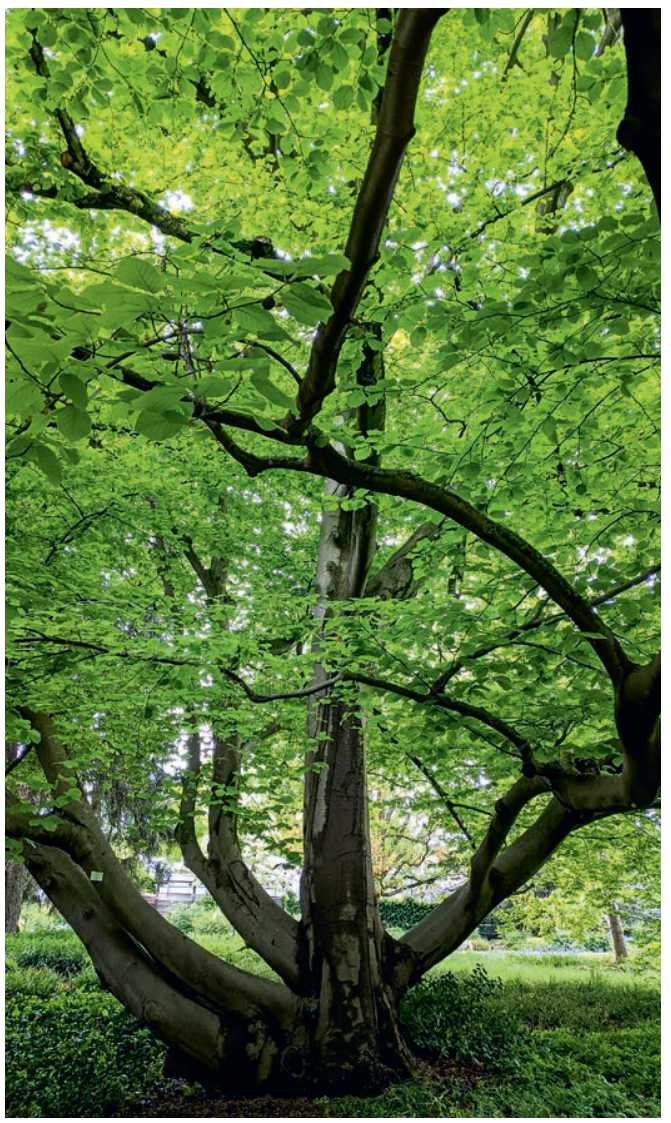

Abb. 6: Gold-Buche im Palmengarten im Frühling.

(Foto: K. Камм)

glatten Rand sind sie gewellt bis leicht gekerbt und zeigen beidseits der Mittelrippe fünf bis acht Seitennerven, die in kurzen Zähnen enden (FELbermeier \& Mosandl 2002, Seybold 2006, StinglWAgner et al. 2009).

Rot-Buchen sind einhäusig getrenntgeschlechtlich, d. h. an einem Baum blühen im April bis Mai sowohl männliche als auch weibliche Blüten. Sie sind unscheinbar, hellgrün und windbestäubt. Die männlichen Blüten hängen zu mehreren in kugeligen Kätzchen herab, während die weiblichen Blüten aufrecht stehen und zu zweit von einer gemeinsamen Hülle umgeben werden, die sich zu einer bestachelten Fruchthülle, der sogenannten Cupula, entwickelt. Im Herbst reifen die typischen, dreikantigen Nussfrüchte der Rot-Buchen zu zweit in dieser Cupula, die sich bei Reife im 


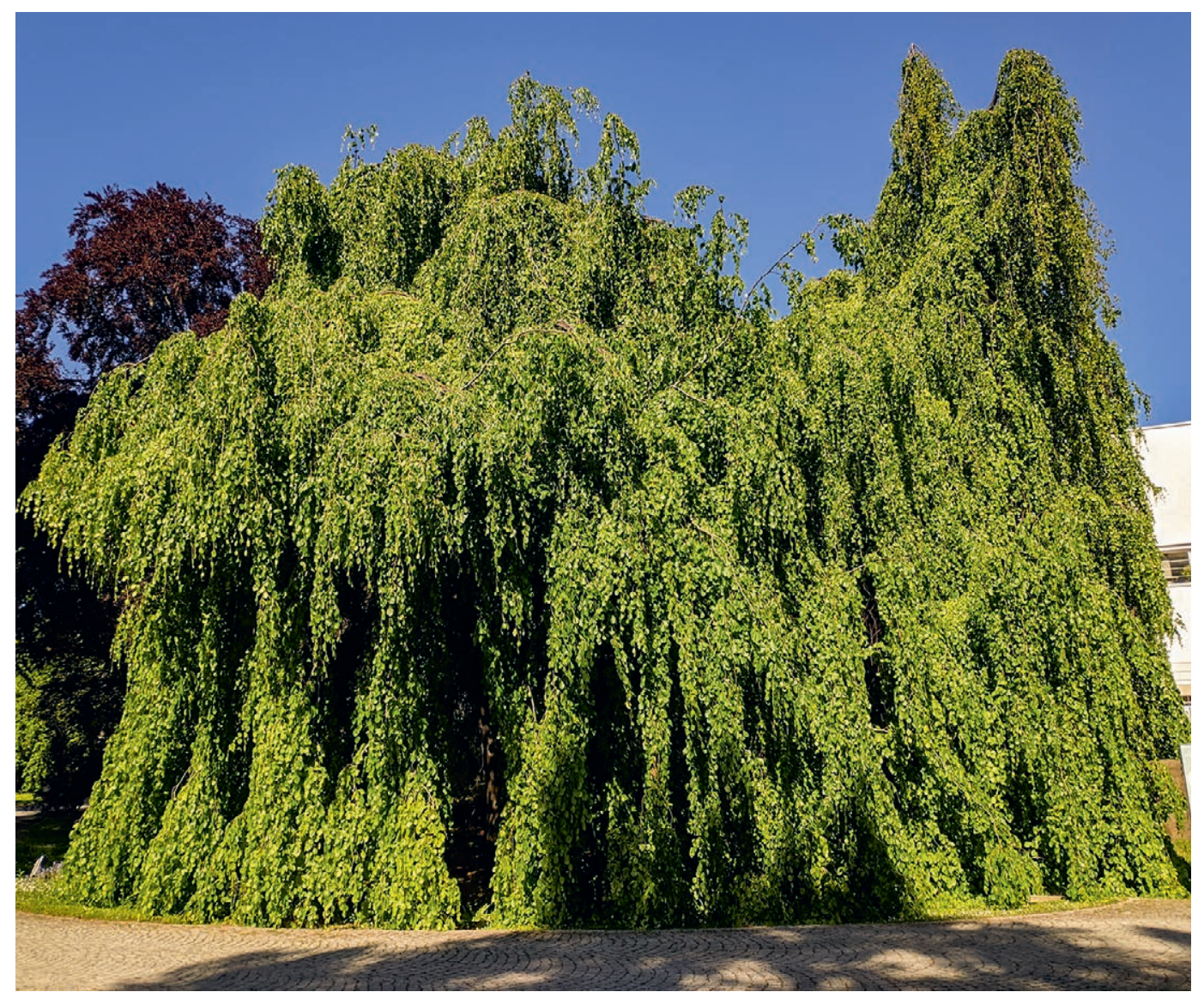

Abb. 7: Die alte Hänge-Buche (Fagus sylvatica 'Pendula') neben dem Gesellschaftshaus in voller Belaubung. (Foto: K. KAMm)

Oktober an vier Nähten öffnet (Felbermeier \& Mosandl 2002, Seybold 2006). Die Früchte der Buche nennt man Bucheckern.

Anhand der Cupula erkennt man auch ihre Verwandtschaft zu Kastanien (Castanea) und Eichen (Quercus), deren Früchte auch von einer ebensolchen Cupula ganz oder teilweise umgeben sind. Zusammen mit den Buchen gehören sie alle zur Familie der Buchengewächse (Fagaceae). Die Gattung Fagus selbst umfasst nur 10 auf der Nordhalbkugel verbreiteten Arten (Stinglwagner et al. 2009). Zu diesen gehören trotz des ähnlichen deutschen Namens weder die heimischen Weiß- bzw. Hainbuchen (Carpinus betulus, Betulaceae), noch die auf der Südhalbkugel heimischen Schein- oder Südbuchen (Nothofagus, Nothofagaceae).

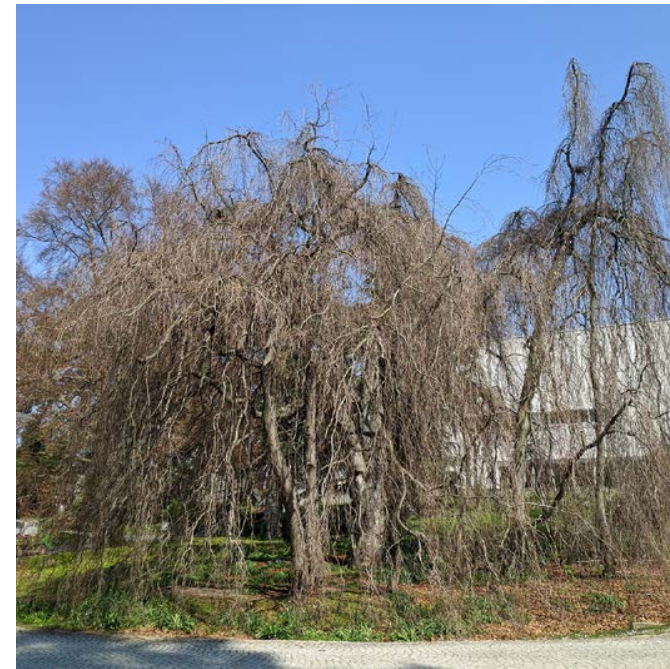

Abb. 8: Die alte Hänge-Buche (Fagus sylvatica 'Pendula') im winterkahlen Zustand. (Foto: K. Kамм) 


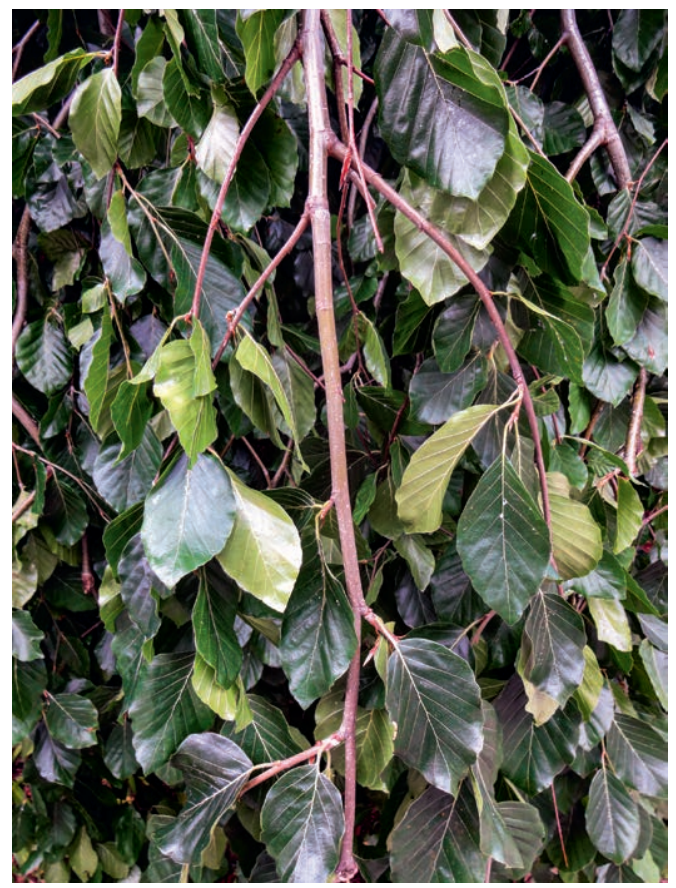

Abb. 9: Hängende Zweige der Hänge-Buche (Fagus sylvatica 'Pendula'). (Foto: K. KAмM)

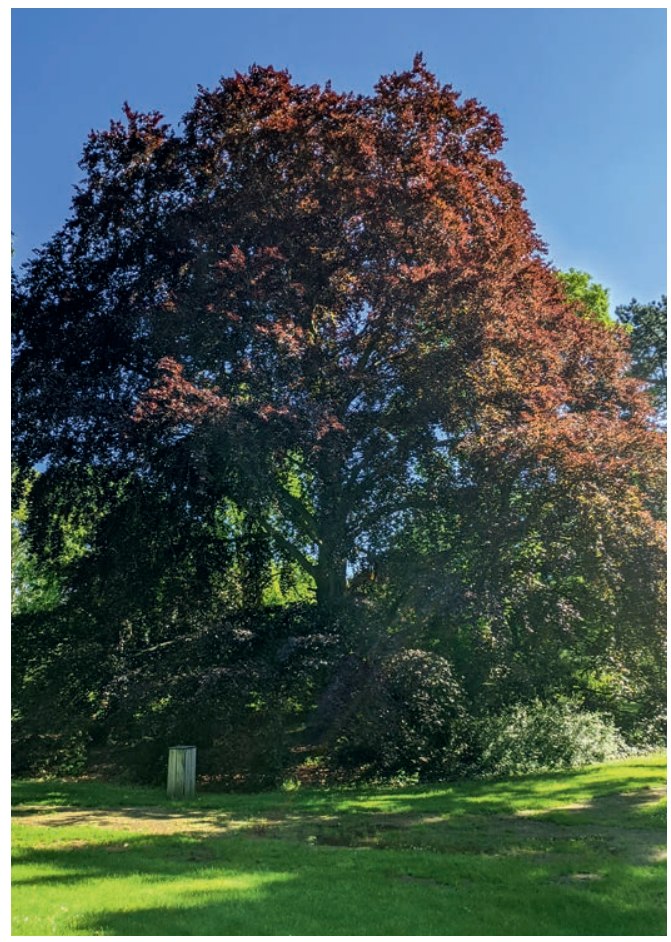

Abb. 10: Blut-Buche an der Liegewiese im Mai. (Foto: K. Камм)

\section{Nutzung}

Die Rot-Buche wird auch als Mutter des Waldes bezeichnet und ist die wichtigste einheimische Laubbaumart (STINGLWAgner et al. 2009). Ihr wertvolles Holz ist rötlich-weiß, manchmal im Alter mit einem sogenannten Rotkern bzw. Falschkern, der durch ein Absinken des Wassergehaltes entsteht (Felbermeier \& Mosandl 2002, StinGLWAGNER et al. 2009). Es ist hart und gut zu bearbeiten, aber wenig elastisch. Es wird beispielsweise zur Herstellung von Möbeln, Spielzeug, Parkett, Furnieren, Sperrholzplatten und Klavieren, aber auch Holzkohle genutzt (STINGLWAGNER et al. 2009, Lieberei \& Reisdorff 2012). Die Wörter Buch und Buchstaben sind von der Buche abgeleitet, da einerseits früher auf Buchenholztafeln geschrieben wurde und andererseits die Germanen Runen in Buchenstäbchen ritzten (STINGLWAGNER et al. 2009).

Aber nicht nur das Holz, auch die Bucheckern können genutzt werden. Sie enthalten bis zu $46 \%$ mildes und gut haltbares Buchelöl. Trotzdem wurden sie nur in Notzeiten gesammelt, da sie von Buchen in großer Menge nur in Mastjahren (d. h. alle 5 bis 15 Jahre) gebildet werden (Lieberei \& Reisdorff 2012) und in Normaljahren gänzlich von Waldbewohnern weggefressen werden können (Felbermeier \& Mosandl 2002). Auch wenn so mancher Wald-Spaziergänger im Herbst gerne Bucheckern nascht, sollte man von einem größeren Verzehr der rohen Bucheckern absehen, da sie u. a. giftige cyanogene Glykoside enthalten (Lieberei \& Reisdorff 2012).

Auch als Ziergehölz werden Rot-Buchen eingesetzt. Neben der ursprünglichen Form sind um die 150 Sorten beschrieben (Felbermeier \& Mosande 2002), die teils sowohl als sortenreine Sämlinge als auch durch Veredelung vermehrt werden. Ihre Klassifizierung ist nicht trivial, da verschiedene Systeme existieren und manche Sorten sich diesen durch eine Kombination von Merkmalen entziehen (CHRISTIAN 2019).

Auch im Palmengarten kommen verschiedene Sorten vor, von denen im Folgenden vier ausgewählte Exemplare vorgestellt werden. Der Ein- 
heitlichkeit und Auffindbarkeit halber werden im Folgenden die Bezeichnungen verwendet, unter denen die Bäume derzeit im Palmengarten gelistet sind.

\subsection{Fagus sylvatica 'Zlatia'}

Am nördlichen Ende des Bambuswäldchens sorgt eine über 25 Meter hohe Fagus sylvatica 'Zlatia' mit ihrem, durch einen früh verzweigenden Stamm gebildeten, weitreichenden Kronendach für reichlich Schatten. Diese 1890 in einem Wald in Serbien gefundene Auslese zeichnet sich durch ihren frühen, fast hell goldgelben Austrieb und die auch im weiteren Jahresverlauf im Vergleich zu Fagus sylvatica helleren Blätter aus (Hertle 1990, FeLBermeier \& Mosandl 2002). 'Zlatia' ist an das serbische Wort für Gold angelehnt, die Sorte wird auch als Gold-Buche bezeichnet.

\subsection{Fagus sylvatica 'Pendula'}

Kaum zu übersehen ist die beeindruckende TrauerBuche (Fagus sylvatica 'Pendula') auf der westlichen Seite des Gesellschaftshauses, direkt nach dem Eingang Palmengartenstraße. Trauer-Buchen haben oft sehr breite, unregelmäßige Kronen (FelberMeier \& Mosandl 2002). Sie werden entweder durch waagerechte Äste gebildet, die nach einer gewissen Länge nach unten herabhängen oder an denen herabhängende Zweige wachsen (РАCKHAM et al. 2012). Die rund 150 Jahre alte Trauer-Buche neben dem Gesellschaftshaus stammt aus den Anfängen des Palmengartens. Sie hat etwa eine Höhe von $20 \mathrm{~m}$ und bildet mit ihrer hängenden Krone eine Art Laube mit einem Durchmesser von etwa 21 Metern. Es verwundert also nicht, dass sie als ein Monumentalbaum Hessens gelistet ist (www. monumentaltrees.com).

\subsection{Fagus sylvatica f. purpurea}

Blut-Buchen verdanken die Rotfärbung ihrer Blätter den im Zellsaft gelösten Pflanzenfarbstoffen, den Anthocyanen. Blut-Buchen waren schon vor 1680 in der Schweiz bekannt (Felbermeier \& Mosandl 2002). Der Legende nach wuchsen fünf Blut-Buchen dort, wo sich fünf Brüder gegenseitig ermordet hatten (CHRIsTIAn 2019). Fast alle Blut-Buchen sollen allerdings von einer ebenfalls Ende des 17. Jahrhunderts entdeckten

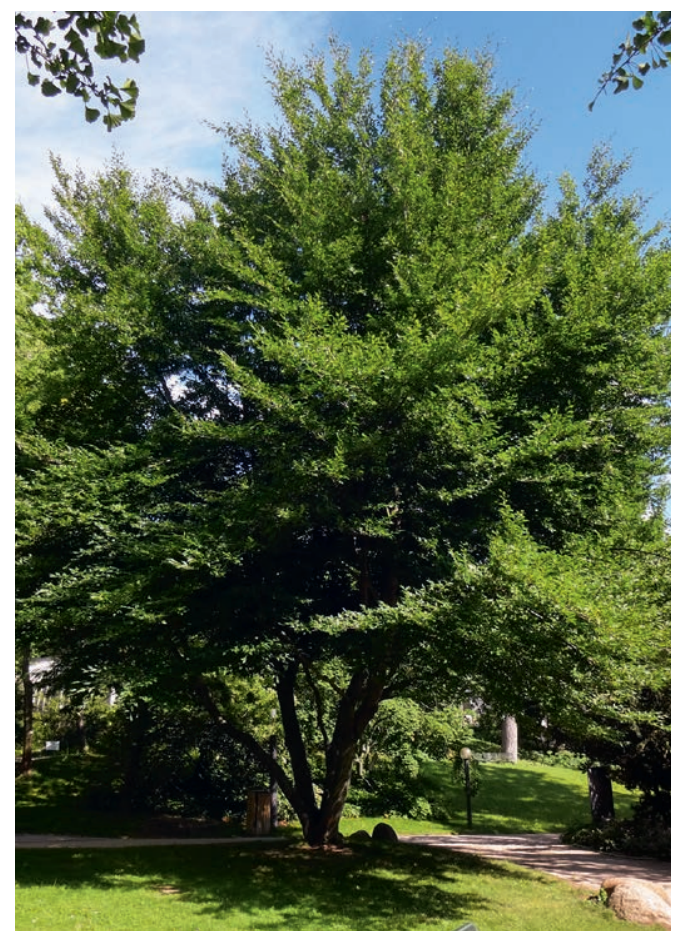

Abb. 11: Farnblättrige Buche Fagus sylvatica 'Asplenifolia'. (Foto: K. Камм)

Blut-Buche im thüringischen Possenwald abstammen (www.kyffhaeuser-nachrichten.de). Es ist wenig überraschend, dass sie im Palmengarten häufiger vorkommen. Stellvertretend soll hier die bemerkenswerte Blut-Buche am südlichen Ende der Liegewiese genannt werden. Sie ist vermutlich über 200 Jahre alt und 34 Meter hoch, bei einem Stammumfang von $4,40 \mathrm{~m}$.

\subsection{Fagus sylvatica 'Asplenifolia'}

Bei dieser Buche weicht die Blattform vom normalen Habitus ab. Asplenium ist der wissenschaftliche Name einer Farn-Gattung (Streifenfarn). Tatsächlich erinnern die schmalen, tief eingeschnittenen Blätter an Farne (Barnard 2002). Wenig überraschend ist daher auch der deutsche Name Farnblättrige Buche. Die Blattform ist allerdings recht variabel, typischerweise werden am Ende eines Triebes fast lanzettförmige Blätter gebildet (Christian 2019). Ein mehrstämmiges Exemplar dieser Varietät steht zwischen dem nördlichen Ende des Palmenhauses und dem Weiher. 


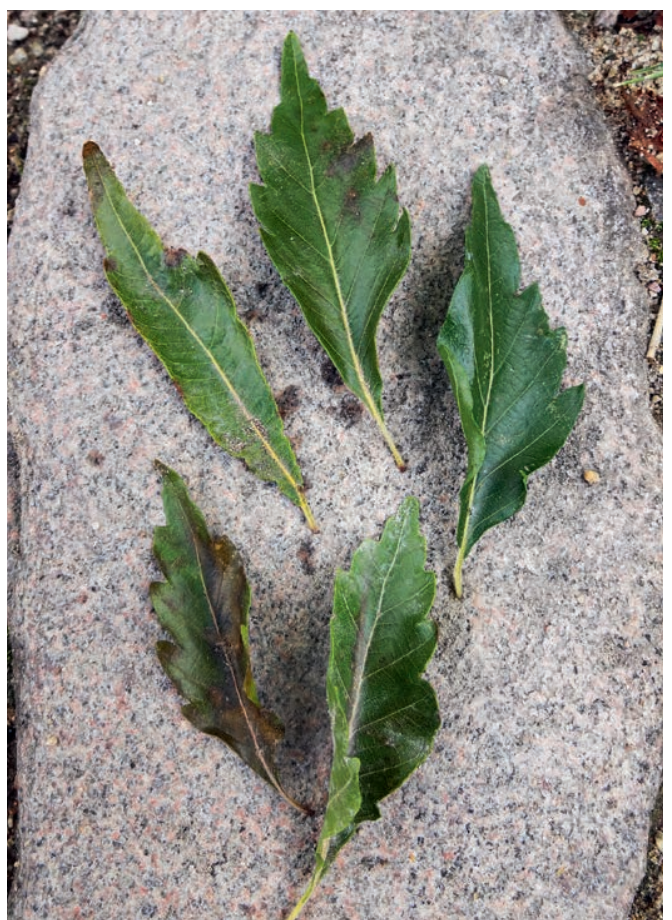

Abbildung 12: Blätter der Farnblättrigen Buche.

(Foto: K. Камм)

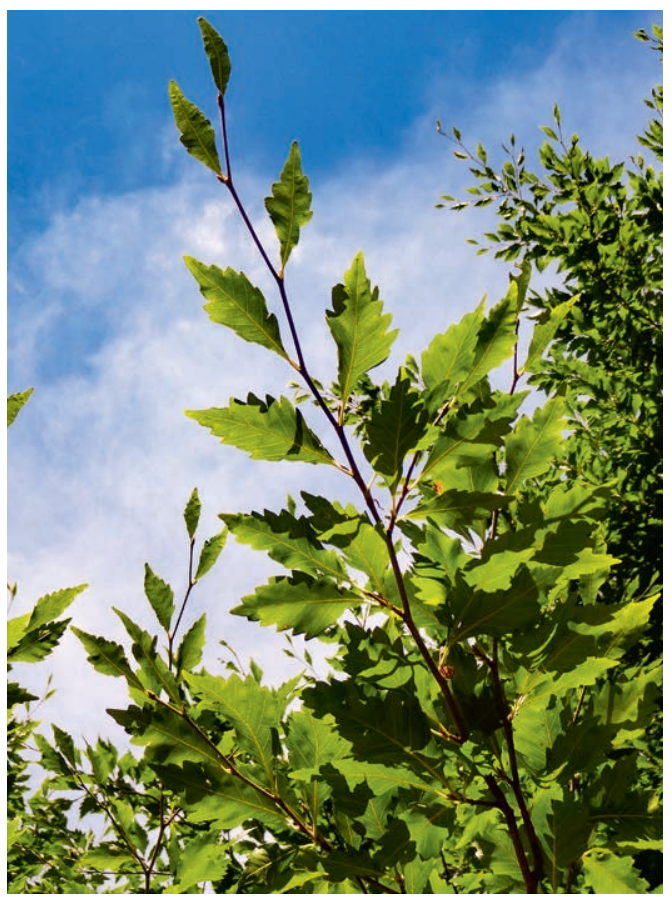

Abb. 13: Belaubter Zweig der Farnblättrigen Buche.

(Foto: K. Камм)
Weitere interessante und besondere Gehölze im Palmengarten lassen sich mit Hilfe des an der Kasse erhältlichen Plans „Sehenswerte Gehölze“ entdecken, aus dem auch die meisten hier zitierten Informationen über die Buchen im Palmengarten stammen.

\section{Literatur}

Bohn, U. \& Neuhäusl, R., unter Mitarbeit von Gollub, G., Hettwer, C., Neuhäuslová, Z., Raus, T., Schlüter, H. \& Weber, H. 2000/2003: Karte der natürlichen Vegetation Europas/Map of the natural vegetation of Europe. Maßstab $1: 2500000$. - Münster.

BARNARD, E.S. 2002: New York city trees, a field guide for the metropolitan area. 3. Aufl. - New York.

Felbermeier, B. \& Mosandl, R. 2002: Fagus sylvatica. In Roloff, A., Weisgerber, H., Lang, U. \& Stimm, B. (Hrsg.) 2010: Bäume Mitteleuropas. - Weinheim.

Hertle, B. 1990: Die Gehölze im Freiland des Palmengartens. - Frankfurt.

Lieberei, R. \& Reisdorff, C. 2012: Nutzpflanzen. 8. Aufl.Stuttgart.

Packham, M, J. R., Thomas, P. A., Atkinson, M. D. \& Degen, T. 2012: Biological flora of the British Isles: Fagus sylvatica. - J. Ecol. 100: 1557-1608

Seybold, S. 2006: Schmeil-Fitschen. Flora von Deutschland und angrenzender Länder. 93. Aufl. - Wiebelsheim.

Stinglwagner, G. K. F., Haseder I. \& Erlebeck, R. 2009: Das Kosmos Wald- und Forstlexikon. 4. Aufl. - Stuttgart.

Wittig, R. \& Streit, B. 2004: Ökologie. - Stuttgart.

\section{Internetseiten:}

http://bwi.info; 77Z1JI_L235of_2012_bi (treesandshrubsonline.org/articles/fagus/fagus-sylvatica/). Accessed 2020-06-09

Christian, T. 2019: „Fagus sylvatica“ from the website trees and shrubs online

https://www.monumentaltrees.com/de/deu/hessen/frankfurt/7249_impalmengarten/14669/

https://www.kyffhaeuser-nachrichten.de/news/news_lang. php?ArtNr=216149

\section{Anschrift der Autorin}

Katharina Kamm, Palmengarten Frankfurt,

Siesmayerstraße 61, 60323 Frankfurt.

E-Mail: Katharina.kamm@stadt-frankfurt.de 